\title{
INCLUSIVE PROMPT PHOTON PRODUCTION IN DEEP INELASTIC SCATTERING AT H1
}

\author{
CARSTEN SCHMITZ \\ ON BEHALF OF THE H1 COLLABORATION \\ University of Zurich \\ Winterthurerstr. 190, 8057 Zurich, Switzerland \\ E-mail: carsten.schmitz@desy.de
}

\begin{abstract}
Results are presented on the inclusive production of isolated prompt photons in deep inelastic scattering with a four-momentum transfer of $Q^{2}>4 \mathrm{GeV}^{2}$. The cross sections are measured for the transverse momentum range of the photons $3<E_{T}^{\gamma}<10 \mathrm{GeV}$ and for the pseudorapidity range of the photons $-1.2<\eta^{\gamma}<$ 1.8. They are measured differentially as a function of $E_{T}^{\gamma}$ and $\eta^{\gamma}$. The results are compared with the predictions of a leading order calculation, which is in reasonable agreement with the inclusive measurement.
\end{abstract}

\section{Introduction}

Isolated photons with high transverse momentum in the final state are a direct probe of the dynamics of the hard subprocess, since they are directly observable without large corrections due to hadronisation and fragmentation. Previously ZEUS and H1 have measured the prompt photon cross section in photoproduction 123 . ZEUS has recently published an analysis of the prompt photon cross section for photon virtualities $Q^{2}$ larger than $35 \mathrm{GeV}^{2} 4$. The present results ${ }^{\mathrm{a}}$ are compared to a leading order calculation $\frac{56}{6}, \mathcal{O}\left(\alpha^{3}\right)$, that offers first predictions for the inclusive prompt photon production in Deep Inelastic Scattering.

\section{Data Sample and Analysis Method}

The events have been collected with the H1-Detector ${ }^{7}$ at HERA in the years $99 / 00$ at a center of mass energy of $318 \mathrm{GeV}$, with a total integrated luminosity of $70.6 \mathrm{pb}^{-1}$.

${ }^{a}$ talk presented at DIS2006 
Events were selected with the electron reconstructed in the backward calorimeter (SpaCa $\left[\frac{8}{8}\right)$. Photons are identified in the H1 liquid argon calorimeter ( $\left.\mathrm{LAl}^{9}\right)$ by a compact electromagnetic cluster with no track pointing to it. To ease the comparison with $\mathrm{pQCD}$ calculations we use an infrared-safe definition of the isolation requirement ${ }^{1011}$ based on the ratio $z$ of the photon energy to the energy of the jet $\frac{12}{12}$ that contains the photon (photonjet).

The photon signal is extracted by a shower shape analysis, which uses six discriminating shower shape functions in a likelihood analysis. The data are corrected for detector effects by taking the average of the corrections of the PYTHIA 6.2 .13 and the HERWIG 6.514 event generator, which model the photon radiation off the quark. Photon radiation off the electron and background from neutral mesons is taken from the RAPGAP 15 generator.

\section{Event Selection}

- DIS Selection: The scattered electron is restricted to the acceptance of the backward calorimeter, $151^{\circ}<\theta_{e}<177^{\circ}$, with an energy $E_{e}^{\prime}$ larger than $10 \mathrm{GeV}$. The four-momentum transfer is furthermore required to be $Q_{e}^{2}>4 \mathrm{GeV}^{2}$ and the inelasticity has to be $y_{e}=1-E_{e}^{\prime}\left(1-\cos \theta_{e}\right) / 2 E_{e}>0.05$.

- Photon Candidate Selection: An electromagnetic cluster is selected with $3<E_{T}^{\gamma}<10 \mathrm{GeV}$ and pseudorapidity $-1.2<\eta^{\gamma}<1.8^{\mathrm{b}}$. No track is allowed to point to the photon candidate within $20 \mathrm{~cm}$.

- Isolation Requirement: the ratio of the photon energy to the energy of the photonjet $z$ has to be larger than 0.9 .

In a first step events are selected with a good electron and a photonjet that contains a photon candidate as defined above. In a second step the prompt photon signal is extracted by a likelihood analysis of shower shapes.

\section{Extraction of the prompt photon signal}

The photon candidate clusters are analysed using six different shower shape variables to discriminate between the signal of a single photon and multiple photons from the decay of neutral mesons.

The estimators are combined in a likelihood analysis, as well as a neural net

\footnotetext{
${ }^{\mathrm{b}}$ The pseudorapidity $\eta$ of a particle with polar angle $\theta$ is given by $\eta=-\ln \tan (\theta / 2)$. $\theta$ is measured with respect to the $z$-axis with the positive axis defined by the direction of the proton, hence positive $\eta$ points in the direction of the proton.
} 
and a range search analysis as a cross check. The likelihood distribution, which provides a considerable separation power, is shown in Figure 1 The data are well described by the sum of simulations. Also the fraction of neutral mesons is well predicted by the unscaled RAPGAP background, which accumulates at low likelihood values.

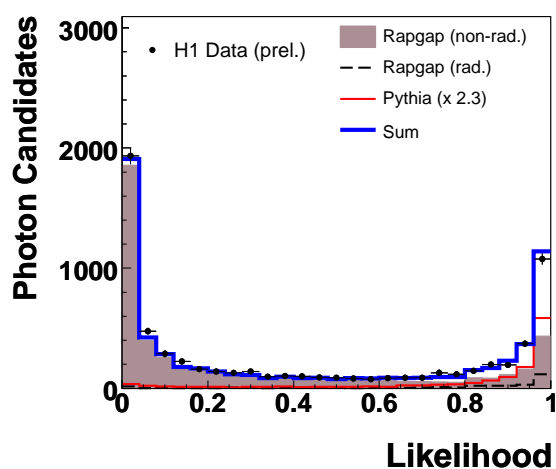

Figure 1. Likelihood Distribution of photon candidates that have passed the primal event selection. The measured data points are shown together with the PYTHIA Monte Carlo (scaled by 2.3), photons radiated off the incoming or outgoing electron (rad) and background from neutral mesons (non-rad) as estimated by RAPGAP. The sum of the Monte Carlo simulations is indicated by the uppermost line.

\section{Results}

Differential cross sections for the production of isolated photons in deep inelastic scattering are presented. Figure 2 shows the comparison with a LO $\left(\alpha^{3}\right)$ calculation 56 . At large pseudorapidities the dominant contribution comes from radiation off the quark line (QQ), whereas in the backward region the radiation off the electron line (LL) dominates the cross section. The calculation slightly underestimates the data.

The data were also compared to the predictions of the PYTHIA and HERWIG generators plus photon radiation off the electron (not shown). Both generators nicely describe the shape in $E_{T}$, but are significantly lower in the abolute scale (factor 2.3 for PYTHIA and 2.6 for HERWIG in order to match the total cross section).

\section{Conclusion}

The data are reasonably described in the covered $\eta^{\gamma}$ and $\mathrm{E}_{T}^{\gamma}$ range by a perturbative LO $\left(\alpha^{3}\right)$ calculation. In addition the data are also described 

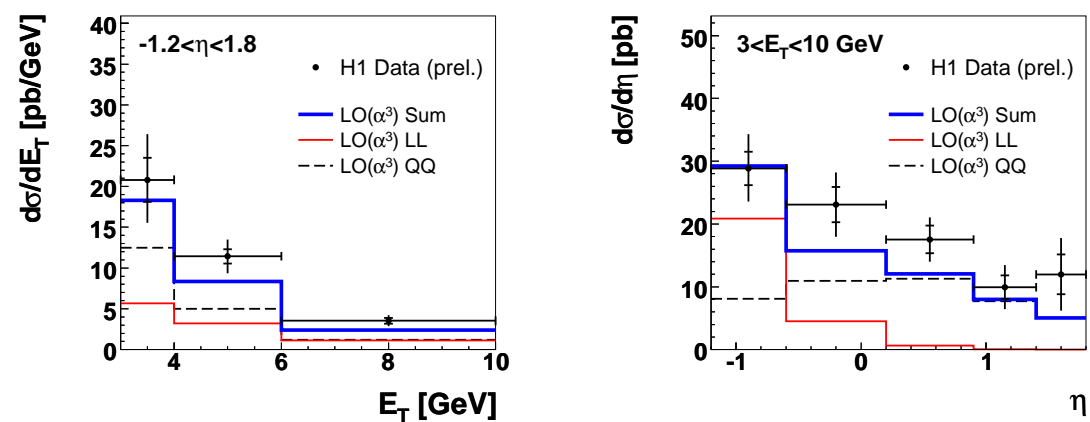

Figure 2. Prompt photon differential cross sections $d \sigma / d E_{T}^{\gamma}$ for $-1.2<\eta^{\gamma}<1.8$ (a) and $d \sigma / d \eta^{\gamma}$ (b) for $3<E_{T}^{\gamma}<10 \mathrm{GeV}$, for photon virtualities $Q^{2}>4 \mathrm{GeV}^{2}$ and $y_{e}>0.05$ compared to a LO calculation. LL and QQ show the contribution of radiation off the electron and the quark line respectively. As the interference is very small it is not shown, but included in the sum.

in shape by the PYTHIA generator plus radiation off the electron line as modelled by RAPGAP, though the absolute scale is too low. The HERWIG generator together with radiative photons shows a somewhat stronger $\eta$ dependence than the data and is also too low in scale.

\section{Acknowledgments}

We would like to thank Aude Gehrmann-de Ridder, Thomas Gehrmann and Eva Poulsen for providing the LO calculations and many fruitful discussions.

\section{References}

1. J. Breitweg et al., Phys. Lett.B 472 (2000) 175 hep-ex/9910045.

2. A. Aktas et al., Eur. Phys. J.C38 (2005) 437 [hep-ex/0407018.

3. E. Brownson, DIS2006 proceedings.

4. S. Chekanov et al., Phys. Lett.B 595 (2004) 86 hep-ex/0402019.

5. A. Gehrmann-De Ridder et al., hep-ph/0601073.

6. A. Gehrmann-De Ridder et al., hep-ph/0604030.

7. I. Abt et al., Nucl. Instr. and Meth. A 386 (1997) 310, ibid, 348.

8. R. D. Appuhn et al., Nucl. Instrum. Meth. A 386 (1997) 397.

9. B. Andrieu et al., Nucl. Instrum. Meth. A336 (1993) 460.

10. E. W. N. Glover and A. G. Morgan, Z. Phys. C 62 (1994) 311.

11. D. Buskulic et al., Z. Phys. C 69 (1996) 365.

12. S. D. Ellis, D. E. Soper, Phys.Rev. D48 (1993) 3160, hep-ph/9305266.

13. T. Sjöstrand et al., hep-ph/0108264.

14. G. Corcella et al., hep-ph/0210213 .

15. H. Jung, Comput. Phys. Commun. 86 (1995) 147. 